\title{
Comparative safety of infliximab and etanercept on the risk of serious infections - Does the association vary by patient characteristics?
}

\author{
Sengwee Toh, $\mathrm{ScD}^{1}$, Lingling $\mathrm{Li}, \mathrm{PhD}^{1}$, Leslie R. Harrold, MD, $\mathrm{MPH}^{2}$, Elizabeth A. Bayliss, \\ MD, MSPH ${ }^{3,4}$, Jeffrey R. Curtis, MD, MS, MPH ${ }^{5}$, Liyan Liu, $\mathbf{M S}^{6}$, Lang Chen, PhD $^{5}$, Carlos G. \\ Grijalva, MD, $\mathrm{MPH}^{7}$, and Lisa J. Herrinton, $\mathrm{PhD}^{6}$ \\ ${ }^{1}$ Department of Population Medicine, Harvard Medical School and Harvard Pilgrim Health Care \\ Institute, Boston, MA, USA \\ ${ }^{2}$ Meyers Primary Care Institute and University of Massachusetts Medical School, Worcester, MA, \\ USA \\ ${ }^{3}$ Institute for Health Research, Kaiser Permanente Colorado, Denver, CO, USA \\ ${ }^{4}$ Department of Family Medicine, University of Colorado School of Medicine, Aurora, CO, USA \\ ${ }^{5}$ Division of Clinical Immunology and Rheumatology, University of Alabama at Birmingham, \\ Birmingham, AL, USA \\ ${ }^{6}$ Division of Research, Kaiser Permanente Northern California, Oakland, CA, USA \\ ${ }^{7}$ Department of Preventive Medicine, Vanderbilt University School of Medicine, Nashville, TN, \\ USA
}

\begin{abstract}
Purpose-Infliximab, a chimeric monoclonal anti-TNF $\alpha$ antibody, has been found to increase the risk of serious infections compared with the TNF receptor fusion protein etanercept in some studies. It is unclear whether the risk varies by patient characteristics. We conducted a study to address this question.
\end{abstract}

Methods-We identified members of Kaiser Permanente Northern California who initiated infliximab $(n=793)$ or etanercept $(n=2,692)$ in 1997-2007. Using a Cox model, we estimated the propensity score-adjusted hazard ratio (HR) and $95 \%$ confidence interval (CI) of serious infections requiring hospitalization or opportunistic infections comparing infliximab with etanercept following treatment initiation. We tested whether the adjusted HR differed by age, sex, race/ ethnicity, body mass index, and smoking status.

Results-The crude incidence rate of serious infections per 100 person-years was 5.4 (95\% CI: $3.8,7.5)$ in patients $<65$ years and $16.0(10.4,23.4)$ in patients $\geq 65$ years during the first three months following treatment initiation. Compared with etanercept, the adjusted HR during this period was elevated for infliximab in patients $<65$ years (HR 3.01; 95\% CI: 1.49, 6.07), but not in those $\geq 65$ years (HR $0.94 ; 0.41,2.13$ ). Findings did not suggest that the HR varied by other patient characteristics examined.

Corresponding author: Sengwee Darren Toh, ScD, Department of Population Medicine, Harvard Medical School and Harvard Pilgrim Health Care Institute, 133 Brookline Ave, $6^{\text {th }}$ Floor, Boston, MA 02215, USA, Tel: +1617509 9818, Fax: +16178598112 , darrentoh@post.harvard.edu.

Conflict of interest: None 
Conclusions-An increased risk of serious infections associated with infliximab relative to etanercept did not appear to be modified by patients' sex, race/ethnicity, body mass index, or smoking status. There was an indication that the increased risk might be limited to patients $<65$ years. Additional studies are warranted to verify or refute this finding.

\section{Keywords}

Anti-TNF agents; Database; Pharmacoepidemiology; Propensity score; Serious infections

\section{Introduction}

Anti-tumor necrosis factor $\alpha(\mathrm{TNF} \alpha)$ agents like etanercept, infliximab, and adalimumab improve clinical, radiographic, and functional outcomes in patients with rheumatoid arthritis (RA). ${ }^{1-6}$ The use of these medications has been expanded to other autoimmune diseases, including psoriatic arthritis, psoriasis, juvenile idiopathic arthritis, ankylosing spondylitis, and inflammatory bowel disease. Although efficacious, anti-TNF $\alpha$ agents have been linked to a number of adverse outcomes, including serious infections. ${ }^{7-14}$ It has been hypothesized that infliximab, a chimeric monoclonal anti-TNF $\alpha$ antibody, might be associated with a greater infection risk relative to the soluble receptor fusion protein etanercept due to their differential effect on the pathophysiology of granulomatous infections. ${ }^{15}$ Results from some studies, particularly those focused on opportunistic infections, are consistent with this hypothesis. ${ }^{13,14,16-22}$

We have previously shown in a large multi-center study that infliximab is associated with a statistically significantly higher risk of serious infections compared with etanercept in RA patients. ${ }^{22}$ Examining the association by specific patient characteristics is a critical next step to better understand the pathophysiology of the differential risk, and generate evidence for better treatment choice and risk management in clinical practice. In the current study, we used data from a subset of that study population for which more detailed individual-level clinical, sociodemographic, and behavioral information (e.g., smoking) was recorded to assess whether the differential risk between infliximab and etanercept is modified by selected patient characteristics.

\section{Methods}

\section{Data source}

This study used data from Kaiser Permanente Northern California (KPNC), a member of the Safety Assessment of Biologic Therapy (SABER) project, a multi-center study jointly funded by the U.S. Agency for Healthcare Research and Quality and the U.S. Food and Drug Administration. ${ }^{23} \mathrm{KPNC}$ is an integrated health care delivery system comprised of a health insurer, insurer-owned hospitals, and a multi-specialty medical group. Its 3.2 million members receive care at one or more of the medical centers of their choosing. Medical care is pre-paid and comprehensive, with nearly all providers being on staff. Referral to rheumatology is through primary care, and only rheumatologists can prescribe biologic agents. KPNC maintains electronic files of eligibility of care and drug coverage; outpatient, emergency department, and inpatient diagnoses and procedures; laboratory testing; and outpatient drug prescriptions for all its members. Its electronic medical record (EMR) system was established in 2004. Before then, several computerized information systems were used to record clinical data. This study was approved by the Institutional Review Board of KPNC. 


\section{Study population}

Among KPNC members aged 17 years or older, we identified patients with a first dispensing of either infliximab or etanercept between January 1, 1997 and December 31, 2007 from the pharmacy dispensing data. We refer to the date of first prescription of either drug as the index date. We required eligible patients to have the following during the 365-day baseline period preceding the index date 1) continuous enrollment and pharmacy benefit, 2) no exposure to any biologic disease-modifying anti-rheumatic drugs (DMARDs), and 3) no recorded diagnosis of serious infections (see below), moderate to severe liver or renal disease, HIV, cancer (except for non-melanoma skin cancer) or solid organ transplantation. We further excluded patients who had a diagnosis of inflammatory bowel disease because etanercept has not been proven effective and not approved for this condition in the U.S. The remaining 793 infliximab and 2,692 etanercept initiators formed our study cohort (Figure 1). For patients with more than one eligible new-use episode, only the first one was analyzed. We did not include adalimumab because it was used as a second-line therapy after failure of either infliximab or etanercept in KPNC during most of the study period. For that reason, patients who received adalimumab in KPNC might be very different from users of the other two anti-TNF $\alpha$ agents with regard to the underlying risk of infection and thus would not be validly comparable.

\section{Outcome}

The outcome of interest was serious infections, defined as infections requiring hospitalization or opportunistic infections. We used the algorithms previously developed in the SABER project ${ }^{22,23}$ to identify serious infection cases. These algorithms have been validated and shown to have positive predictive values of $80 \%$ or greater. ${ }^{12,24,25}$

\section{Potential confounders and effect modifiers}

We identified the following potential confounders and effect modifiers from the 365-day baseline period: age; sex; race/ethnicity; body mass index (BMI); smoking status; type of insurance; the proportion of household below the poverty line in the census block in which the patient lived (as a proxy for socioeconomic status), Charlson comorbidity score; diagnosis of autoimmune diseases (RA, psoriatic arthritis, psoriasis, ankylosing spondylitis), diabetes, or chronic obstructive pulmonary disease; use of methotrexate, hydroxychloroquine, leflunomide, sulfasalazine, non-steroidal anti-inflammatory drugs, opioids, corticosteroids, or antibiotics (for non serious infections or other conditions); and the number of inpatient visits, outpatient visits, and unique medications dispensed (Table 1).

We extracted information on patient age, sex, race/ethnicity and type of insurance from KPNC's administrative files. We obtained the proportion of household below the poverty line of the census block from the linked census data. Electronic clinical data provided information on diagnoses, medication use, health services utilization, and Charlson comorbidity score (derived from specific International Classification of Diseases, $9^{\text {th }}$ Revision, Clinical Modification [ICD-9-CM] $\operatorname{codes}^{26}$ ).

For 3,146 (90\%) of the study patients, race/ethnicity was available from the health plan's satisfaction surveys, large self-administered member health surveys, or mortality and hospitalization data. We used these sources in this order so that self-reported information was given precedence over provider-recorded information. Baseline BMI information was available from EMRs for 1,800 (52\%) of the study patients. For the remaining patients, specific ICD-9-CM codes were used to determine BMI; patients who had an ICD-9-CM code for overweight or obesity were classified as such, and patients without these codes were defined as normal/underweight otherwise. Smoking status was determined at baseline from EMRs for 1,190 (34\%) of the study patients. ICD-9-CM codes were used for the 
remaining patients. Using data from patients whose BMI or smoking data were recorded in EMRs, we were able to verify the high accuracy of the ICD-9-CM codes (Appendix).

\section{Statistical analysis}

Follow-up of each patient started on the index date, and ended at the earliest occurrence of the first serious infection, death, health plan disenrollment, December 31, 2007, or 365 days after the index date. We chose a maximal 365-day follow-up period because previous studies suggested that serious infection risk was the highest immediately following treatment initiation, usually during the first 3 to 6 months, ${ }^{10,12,13,18}$ and we were interested in the short- and intermediate-term risk associated with anti-TNFa therapy. We calculated the incidence rate and Poisson 95\% confidence interval (CI) of serious infections among initiators of infliximab and etanercept, for all patients and by specific subgroup (see below).

We used propensity score 27,28 to adjust for potential confounders. The propensity score was the probability of initiating infliximab compared with etanercept, we estimated it through a logistic model that included all the potential confounders described above. We employed an "intention-to-treat" approach to compare the risk of serious infections between infliximab initiators and etanercept initiators. This analysis assessed the effect of treatment initiation, ignoring whether the patients continued the initial therapy during follow-up. We fit a Cox model that included an indicator for the exposure and the propensity score (in quintiles) to estimate the hazard ratio (HR) and $95 \%$ CI. The time scale was time since the index date. We estimated the average HRs and 95\% CIs for 0-3, 0-6, and 0-12 months after treatment initiation.

To estimate the subgroup-specific HRs and $95 \%$ CIs, we stratified the analysis by age ( $<65$ vs. $\geq 65$ years), sex (male vs. female), race/ethnicity (Non-Hispanic White, AfricanAmerican, Hispanic, Asian American, Native American, and other/unknown), BMI ( $<25$ vs. $\geq 25 \mathrm{~kg} / \mathrm{m}^{2}$ ), and smoking status (non, past, and current smoker). We performed formal statistical tests to examine if the relation between anti-TNF $\alpha$ therapy and serious infections varied by these five patient characteristics. For each potential effect modifier, we fit two Cox models using the entire study cohort - one with and one without additional interaction terms between anti-TNF $\alpha$ therapy and the variable. We then conducted a likelihood ratio test $^{29}$ to assess the statistical significance of the interaction terms at the 0.05 level. Because this was not a confirmatory study, we did not adjust for multiple comparisons.

\section{Sensitivity analysis}

Although the intention-to-treat analysis preserves baseline comparability of the two groups conditional on the propensity score, the analysis may be progressively biased over time with increasing misclassification of exposure due to treatment switching or discontinuation. We performed a secondary analysis that censored patients when they stopped their initial treatment. In doing so, we combined consecutive dispensings of the same drug for each patient to create a continuous drug use period that began on the index date. We allowed a grace-period of 30 days for etanercept and 60 days for infliximab between two consecutive dispensings, because etanercept is self-administered via injection (each injection covers seven days and each dispensing containing four injections for a 28-day supply at KPNC) while infliximab is administered through infusion (each infusion covers 56 days at KPNC). The secondary analysis censored patients when their continuous drug use period ended. This "as-treated" analysis examined the effect of actual drug use, but might introduce selection bias if patients who adhered to their initial treatment had a different risk profile than those who switched or stopped therapy during follow-up. ${ }^{30,31}$ 
We used a stepwise regression to identify potentially important two-way interaction terms between each of the five patient characteristics and other potential confounders in building the propensity score model. Finally, we performed an analysis restricting to patients with known BMI and smoking information in EMRs, and to RA patients.

\section{Results}

\section{Patient characteristics}

Of the 3,485 study patients, $19 \%$ were 65 years or older, $65 \%$ were female, $42 \%$ had a BMI of $25 \mathrm{~kg} / \mathrm{m}^{2}$ or greater, and $18 \%$ were current smokers. The patients were racially and ethnically diverse: $66 \%$ were Non-Hispanic White, $5 \%$ were African American, $6 \%$ were Hispanic, $11 \%$ were Asian American, 2\% were Native American, and 10\% had other or unknown race/ethnicity information. There were only four cases and one case, respectively, in Hispanics and the other/unknown group, and we were unable to translate the findings from patients with the other/unknown group into actionable information. Therefore, data from these patients were excluded from the analysis that examined effect modification by race/ethnicity. Table 1 shows the association between each confounder and initiation of infliximab estimated from the propensity score model, which had a c-statistic of 0.79 .

\section{Crude incidence rate of serious infections}

The average follow-up was 335 days in infliximab initiators and 328 days in etanercept initiators. One hundred and seventy six patients (5\%) developed a serious infection. The incidence rate per 100 person-years in all patients was 7.5 during $0-3$ months, 6.6 during 0 6 months, and 5.6 during $0-12$ months following treatment initiation (Table 2). The incidence rate was approximately 3 -folder higher among patients aged $\geq 65$ years than among patients $<65$ years. Female, Native Americans and African Americans, and patients with BMI $<25 \mathrm{~kg} / \mathrm{m}^{2}$ had a greater incidence rate. The incidence rate was comparable between non smokers and current smokers; past smokers had the highest incidence rate.

\section{Relative risk of serious infections, all patients}

The risk of serious infections was higher in infliximab initiators than in etanercept initiators (Table 3). In general, the HRs attenuated upon adjustment for potential confounders. The increased risk associated with infliximab was the greatest during the first three months after treatment initiation (adjusted HR 1.91; 95\% CI: 1.10, 3.34). The risk remained elevated but diminished over time: the adjusted HR was $1.64(1.07,2.52)$ in $0-6$ months, and $1.13(0.80$, $1.60)$ in $0-12$ months.

\section{Relative risk of serious infections, by age, sex, and race/ethnicity}

The time-dependent increased risk of serious infections associated with infliximab was observed in patients $<65$ years old, but not in those $\geq 65$ years (Table 3). Formal statistical tests for effect modification by age group were not statistically significant, although the pvalue was 0.06 in $0-3$ months. There was no indication of effect modification by sex. The tests for effect modification by race/ethnicity were not statistically significant (Table 4).

\section{Relative risk of serious infections, by BMI and smoking status}

Although the adjusted HRs of serious infections were greater in patients with BMI $<25 \mathrm{~kg}$ / $\mathrm{m}^{2}$ across different follow-up periods, the tests for effect modification by BMI were not statistically significant (Table 5). Excluding patients who were underweight (BMI $<18.5 \mathrm{~kg} /$ $\mathrm{m}^{2}$ ) (six cases of serious infections) did not change the results. The adjusted HR also seemed to be different across smoking statuses, but the pattern was not uniform over time and the differences did not reach statistical significance. 


\section{Sensitivity analysis}

The adjusted HRs were similar but slightly higher in the as-treated analysis. For example, the adjusted HR was $2.38(1.28,4.43)$ in patients aged $<65$ years and $1.15(0.56,2.38)$ in those aged $\geq 65$ years in $0-6$ months following treatment initiation. The stratum-specific estimates were qualitatively similar with wider $95 \%$ CIs when we restricted the analyses to patients whose BMI or smoking information was available in EMRs. For example, the adjusted HR was $3.82(1.60,9.17)$ in patients aged $<65$ years and $1.15(0.41,3.21)$ in those aged $\geq 65$ years in $0-6$ months following treatment initiation. Results (not shown) from the stepwise regression analysis were similar.

Restricting the analyses to RA patients did not materially change the results. The adjusted HR was $2.16(1.19,3.93)$ in $0-3$ months, $1.45(0.90,2.32)$ in $0-6$ months, and $1.02(0.69$, $1.51)$ in $0-12$ months. Among patients aged $<65$ years, the adjusted HR was 3.83 (1.79, $8.19)$ in $0-3$ months, $1.95(1.02,3.71)$ in $0-6$ months, and $1.18(0.67,2.09)$ in $0-12$ months. Among patients aged $\geq 65$ years, the corresponding HR was $0.99(0.41,2.39), 1.07(0.55$, $2.11)$, and $0.91(0.53,1.56)$.

\section{Discussion}

In this study, we found a time-dependent, higher risk of serious infections among infliximab initiators compared with etanercept initiators. The elevated risk associated with infliximab initiation appeared to vary by patient age, with an increased risk observed in patients aged $<65$ years, but not in those $\geq 65$ years of age.

Infliximab is a chimeric monoclonal antibody targeting TNF $\alpha$ while etanercept is a soluble $\mathrm{TNF} \alpha$ receptor. It has been hypothesized that infliximab and etanercept might have differential effects on granulomatous inflammatory conditions. ${ }^{17,32}$ Specifically, infliximab, but not etanercept, appears to disrupt established granulomas and increase infection risk of granulomatous diseases through 1) greater inhibition of TNF signaling events, 2) more complete blockade of TNF activity, 3) greater induction of apoptotic activity within granulomas, or 4) a combination of these pathways. ${ }^{17}$ This hypothesis is consistent with findings from a number of population-based observational studies. ${ }^{13,14,16-22}$ To our knowledge, this is the first study specifically designed to examine whether the differential risk of serious infections between the two anti-TNF $\alpha$ agents varies by important modifiable and non-modifiable patient characteristics.

Older age is a strong risk factor for acquiring infections in the general population ${ }^{33,34}$ and in RA patients. ${ }^{10,12,14,35-38}$ In our study, infliximab initiators were more likely to be aged $\geq 65$ years, which might be due to a more generous coverage of the drug in Medicare. ${ }^{39} \mathrm{We}$ found that patients $\geq 65$ years had an approximately 3 -fold higher incidence rate of serious infections than patients $<65$ years. Compared with initiation of etanercept, an increased risk associated with infliximab initiation was only observed in patients $<65$ years. We hypothesize that this may be due to a "ceiling effect": the underlying risk was already high in older patients due to the presence of various risk factors, the effect of an additional risk factor (i.e., infliximab) may therefore be less pronounced when measured on a relative scale in these patients than in those without other risk factors. Alternatively, older age may be a proxy for other factors that modify the observed effect. Our finding may also be due to chance.

There was no strong evidence to suggest that the effect of infliximab on serious infections varied by sex, race/ethnicity, BMI, or smoking status. As shown in Table 2, the underlying risk at each sex, current smoking (but not past smoking), and BMI stratum was likely not 
great enough to lead to a ceiling effect. Although the incidence rate in Native Americans and African Americans was high, the estimate was based on a small number of cases.

Smoking is a risk factor for infection. ${ }^{40,41}$ Some have observed that RA patients who smoked might have a higher serious infection risk, ${ }^{36}$ but this finding was not found in other studies. ${ }^{35,38}$ The high incidence rate in past smokers is intriguing. A possible explanation is that patients who were at a higher risk of developing a serious infection might be more likely to stop smoking prior to the initiation of anti-TNF $\alpha$ therapy given the perceived infection risk associated with these medications. Alternatively, patients might quit smoking due to symptoms from the lung damage caused by smoking, which puts them at an increased risk of acquiring a serious infection. However, the precise timing of smoking cessation was not available in our cohort, limiting our ability to further investigate this issue and might have led to misclassification of smoking status. Alternatively, the results might be due to random error as the number of cases was small among past smokers.

Our study population was community-based, well characterized, and diverse. The depth of our database allowed us to adjust for multiple confounders and examine several effect modifiers that are often not available in other databases. We directly compared two anti$\mathrm{TNF} \alpha$ agents, thereby reducing confounding by underlying disease severity and potential bias introduced by disparate infection risk management between users of anti-TNF $\alpha$ drugs and non-biologic DMARDs.

On the other hand, the results should be interpreted in the context of the limitations of the study. As we did not have direct measures of disease severity, there might be residual confounding. However, it is reassuring that our results were consistent with other studies that had information on disease severity. ${ }^{14,18,20}$ We used a propensity score developed for the entire cohort for all analyses. It is possible that the propensity score might not perform equally well in all subgroups. A more ideal approach would be to estimate subgroup-specific propensity score, but limited sample size precluded us from doing so. In a sensitivity analysis, we used an alternative approach that included in the propensity score model potentially important interaction terms identified through a stepwise regression approach, and the results were similar.

The intention-to-treat analysis might be biased due to misclassification of exposure during follow-up, whereas the as-treated analysis might introduce bias due to selection of patients who were adherent to their initial therapy. ${ }^{30,31}$ However, it is reassuring that both analyses provided consistent results. As BMI and smoking status were determined partly from ICD-9CM codes (48\% for BMI and 66\% for smoking), some patients might have been misclassified. However, restricting the analyses to patients whose BMI and smoking information were available in EMRs provided qualitatively similar results. Finally, the number of cases in certain subgroups was small, therefore the statistical power to detect an effect modification might be limited, and some findings might have been due to chance.

In conclusion, our study suggested that the higher risk of serious infections associated with infliximab initiation compared with etanercept initiation did not appear to be modified by patients' sex, race/ethnicity, BMI, or smoking status. There was an indication that the increased risk might be limited to patients younger than 65 years. More studies are needed to verify or refute our findings.

\section{Acknowledgments}

Source of financial support: This study was supported by grant 1U18HS016955 from the Agency for Healthcare Research and Quality to the HMO Research Network Center for Education and Research in Therapeutics (CERT). 
Dr. Harrold is supported by NIH (AR053856), Dr. Curtis is supported by NIH (AR053351), and Dr. Grijalva is supported by NIH (P60AR056116).

The content is solely the responsibility of the authors and does not necessarily represent the official views of the Agency for Healthcare Research Quality. The authors acknowledge the tools and resources developed under the Agency for Health Research and Quality cooperative agreement number 1U18H17919. The authors also thank Dr. Kevin Winthrop at OHSU and Dr. John Baddley at UAB for leading the SABER workgroup group that created the definition of serious infections.

\section{References}

1. Maini R, St Clair EW, Breedveld F, et al. Infliximab (chimeric anti-tumour necrosis factor alpha monoclonal antibody) versus placebo in rheumatoid arthritis patients receiving concomitant methotrexate: a randomised phase III trial. ATTRACT Study Group. Lancet. 1999; 354:1932-1939. [PubMed: 10622295]

2. Lipsky PE, van der Heijde DM, St Clair EW, et al. Infliximab and methotrexate in the treatment of rheumatoid arthritis. Anti-Tumor Necrosis Factor Trial in Rheumatoid Arthritis with Concomitant Therapy Study Group. N Engl J Med. 2000; 343:1594-1602. [PubMed: 11096166]

3. Moreland LW, Schiff MH, Baumgartner SW, et al. Etanercept therapy in rheumatoid arthritis. A randomized, controlled trial. Ann Intern Med. 1999; 130:478-486. [PubMed: 10075615]

4. Genovese MC, Bathon JM, Martin RW, et al. Etanercept versus methotrexate in patients with early rheumatoid arthritis: two-year radiographic and clinical outcomes. Arthritis Rheum. 2002; 46:14431450. [PubMed: 12115173]

5. Keystone EC, Kavanaugh AF, Sharp JT, et al. Radiographic, clinical, and functional outcomes of treatment with adalimumab (a human anti-tumor necrosis factor monoclonal antibody) in patients with active rheumatoid arthritis receiving concomitant methotrexate therapy: a randomized, placebo-controlled, 52-week trial. Arthritis Rheum. 2004; 50:1400-1411. [PubMed: 15146409]

6. Breedveld FC, Weisman MH, Kavanaugh AF, et al. The PREMIER study: A multicenter, randomized, double-blind clinical trial of combination therapy with adalimumab plus methotrexate versus methotrexate alone or adalimumab alone in patients with early, aggressive rheumatoid arthritis who had not had previous methotrexate treatment. Arthritis Rheum. 2006; 54:26-37. [PubMed: 16385520]

7. Listing J, Strangfeld A, Kary S, et al. Infections in patients with rheumatoid arthritis treated with biologic agents. Arthritis Rheum. 2005; 52:3403-3412. [PubMed: 16255017]

8. Bongartz T, Sutton AJ, Sweeting MJ, et al. Anti-TNF antibody therapy in rheumatoid arthritis and the risk of serious infections and malignancies: systematic review and meta-analysis of rare harmful effects in randomized controlled trials. JAMA. 2006; 295:2275-2285. [PubMed: 16705109]

9. Bernatsky S, Hudson M, Suissa S. Anti-rheumatic drug use and risk of serious infections in rheumatoid arthritis. Rheumatology (Oxford). 2007; 46:1157-1160. [PubMed: 17478469]

10. Askling J, Fored CM, Brandt L, et al. Time-dependent increase in risk of hospitalisation with infection among Swedish RA patients treated with TNF antagonists. Ann Rheum Dis. 2007; 66:1339-1344. [PubMed: 17261532]

11. Askling J, Dixon W. The safety of anti-tumour necrosis factor therapy in rheumatoid arthritis. Curr Opin Rheumatol. 2008; 20:138-144. [PubMed: 18349742]

12. Curtis JR, Patkar N, Xie A, et al. Risk of serious bacterial infections among rheumatoid arthritis patients exposed to tumor necrosis factor alpha antagonists. Arthritis Rheum. 2007; 56:1125-1133. [PubMed: 17393394]

13. Curtis JR, Xi J, Patkar N, et al. Drug-specific and time-dependent risks of bacterial infection among patients with rheumatoid arthritis who were exposed to tumor necrosis factor alpha antagonists. Arthritis Rheum. 2007; 56:4226-4227. [PubMed: 18050253]

14. Strangfeld A, Listing J, Herzer P, et al. Risk of herpes zoster in patients with rheumatoid arthritis treated with anti-TNF-alpha agents. JAMA. 2009; 301:737-744. [PubMed: 19224750]

15. Wallis RS, Ehlers S. Tumor necrosis factor and granuloma biology: explaining the differential infection risk of etanercept and infliximab. Semin Arthritis Rheum. 2005; 34:34-38. [PubMed: 15852254] 
16. Keane J, Gershon S, Wise RP, et al. Tuberculosis associated with infliximab, a tumor necrosis factor alpha-neutralizing agent. N Engl J Med. 2001; 345:1098-1104. [PubMed: 11596589]

17. Wallis RS, Broder MS, Wong JY, et al. Granulomatous infectious diseases associated with tumor necrosis factor antagonists. Clin Infect Dis. 2004; 38:1261-1265. [PubMed: 15127338]

18. Dixon WG, Symmons DP, Lunt M, et al. Serious infection following anti-tumor necrosis factor alpha therapy in patients with rheumatoid arthritis: lessons from interpreting data from observational studies. Arthritis Rheum. 2007; 56:2896-2904. [PubMed: 17763441]

19. Salliot C, Gossec L, Ruyssen-Witrand A, et al. Infections during tumour necrosis factor-alpha blocker therapy for rheumatic diseases in daily practice: a systematic retrospective study of 709 patients. Rheumatology (Oxford). 2007; 46:327-334. [PubMed: 16880188]

20. Favalli EG, Desiati F, Atzeni F, et al. Serious infections during anti-TNFalpha treatment in rheumatoid arthritis patients. Autoimmun Rev. 2009; 8:266-273. [PubMed: 19022409]

21. Curtis JR, Xie F, Chen L, et al. The comparative risk of serious infections among rheumatoid arthritis patients starting or switching biological agents. Ann Rheum Dis. 2011; 70:1401-1406. [PubMed: 21586439]

22. Grijalva CG, Chen L, Delzell E, et al. Initiation of tumor necrosis factor-alpha antagonists and the risk of hospitalization for infection in patients with autoimmune diseases. JAMA. 2011; 306:23312339. [PubMed: 22056398]

23. Herrinton LJ, Curtis JR, Chen L, et al. Study design for a comprehensive assessment of biologic safety using multiple healthcare data systems. Pharmacoepidemiol Drug Saf. 2011; 20:1199-1209. [PubMed: 21919113]

24. Patkar NM, Curtis JR, Teng GG, et al. Administrative codes combined with medical records based criteria accurately identified bacterial infections among rheumatoid arthritis patients. J Clin Epidemiol. 2009; 62:321-327. 327, e321-327. [PubMed: 18834713]

25. Schneeweiss S, Robicsek A, Scranton R, et al. Veteran's affairs hospital discharge databases coded serious bacterial infections accurately. J Clin Epidemiol. 2007; 60:397-409. [PubMed: 17346615]

26. Deyo RA, Cherkin DC, Ciol MA. Adapting a clinical comorbidity index for use with ICD-9-CM administrative databases. J Clin Epidemiol. 1992; 45:613-619. [PubMed: 1607900]

27. Rosenbaum PR, Rubin DB. The central role of the propensity score in observational studies for causal effects. Biometrika. 1983; 70:41-55.

28. Rosenbaum PR, Rubin DB. Reducing bias in observational studies using subclassification on the propensity score. J Am Stat Assoc. 1984; 79:516-524.

29. Prentice R. Use of the logistic model in retrospective studies. Biometrics. 1976; 32:599-606. [PubMed: 963173]

30. Hernán MA, Hernandez-Diaz S, Robins JM. A structural approach to selection bias. Epidemiology. 2004; 15:615-625. [PubMed: 15308962]

31. Toh S, Hernán MA. Causal inference from longitudinal studies with baseline randomization. Int J Biostat. 2008; 4:Article22.

32. Marino S, Sud D, Plessner H, et al. Differences in reactivation of tuberculosis induced from antiTNF treatments are based on bioavailability in granulomatous tissue. PLoS Comput Biol. 2007; 3:1909-1924. [PubMed: 17953477]

33. Gardner ID. The effect of aging on susceptibility to infection. Rev Infect Dis. 1980; 2:801-810. [PubMed: 6763306]

34. Gavazzi G, Krause KH. Ageing and infection. Lancet Infect Dis. 2002; 2:659-666. [PubMed: 12409046]

35. Dixon WG, Watson K, Lunt M, et al. Rates of serious infection, including site-specific and bacterial intracellular infection, in rheumatoid arthritis patients receiving anti-tumor necrosis factor therapy: results from the British Society for Rheumatology Biologics Register. Arthritis Rheum. 2006; 54:2368-2376. [PubMed: 16868999]

36. Doran MF, Crowson CS, Pond GR, et al. Predictors of infection in rheumatoid arthritis. Arthritis Rheum. 2002; 46:2294-2300. [PubMed: 12355476]

37. Wolfe F, Caplan L, Michaud K. Treatment for rheumatoid arthritis and the risk of hospitalization for pneumonia: associations with prednisone, disease-modifying antirheumatic drugs, and antitumor necrosis factor therapy. Arthritis Rheum. 2006; 54:628-634. [PubMed: 16447241] 
38. Wolfe F, Michaud K, Chakravarty EF. Rates and predictors of herpes zoster in patients with rheumatoid arthritis and non-inflammatory musculoskeletal disorders. Rheumatology (Oxford). 2006; 45:1370-1375. [PubMed: 17003175]

39. Doshi JA, Li P, Puig A. Impact of the Medicare Modernization Act of 2003 on utilization and spending for medicare part B-covered biologics in rheumatoid arthritis. Arthritis Care Res (Hoboken). 2010; 62:354-361. [PubMed: 20391481]

40. Sherman CB. The health consequences of cigarette smoking. Pulmonary diseases. Med Clin North Am. 1992; 76:355-375. [PubMed: 1548966]

41. Almirall J, Gonzalez CA, Balanzo X, et al. Proportion of community-acquired pneumonia cases attributable to tobacco smoking. Chest. 1999; 116:375-379. [PubMed: 10453865]

\section{Appendix. Accuracy of body mass index and smoking information obtained from ICD-9-CM codes using information recorded in electronic medical records (EMRs) as the gold standard, Kaiser Permanente Northern California, 1997-2007}

\begin{tabular}{|l|c|c|c|c|}
\hline \multicolumn{4}{|c|}{ Body mass index $\left(\mathbf{k g} / \mathbf{m}^{2}\right)$} \\
\hline \multirow{2}{*}{ ICD-9-CM } & \multicolumn{4}{|c|}{ Electronic medical records } \\
\cline { 2 - 5 } & $<\mathbf{2 5}$ & $\mathbf{2 5 - 2 9}$ & $\mathbf{2 3 0}$ & Total \\
\hline$<25$ & 385 & 162 & 156 & 703 \\
\hline $25-29$ & 61 & 374 & 6 & 441 \\
\hline$\geq 30$ & 8 & 61 & 587 & 656 \\
\hline Total & 454 & 597 & 749 & 1,800 \\
\hline
\end{tabular}

Positive predictive value

$25-29 \mathrm{~kg} / \mathrm{m}^{2}: 374 / 441=85 \%$

$\geq 30 \mathrm{~kg} / \mathrm{m}^{2}: 587 / 656=89 \%$

"Negative" predictive value

$<25 \mathrm{~kg} / \mathrm{m}^{2}: 385 / 703=55 \%$

\section{Sensitivity}

$<25 \mathrm{~kg} / \mathrm{m}^{2}: 385 / 454=85 \%$

$25-29 \mathrm{~kg} / \mathrm{m}^{2}: 374 / 597=63 \%$

$\geq 30 \mathrm{~kg} / \mathrm{m}^{2}: 587 / 749=78 \%$

\begin{tabular}{|l|c|c|c|c|}
\hline Smoking status \\
\hline \multirow{2}{*}{ ICD-9-CM } & \multicolumn{4}{|c|}{ Electronic medical records } \\
\cline { 2 - 5 } & Non smoker & Past smoker & Current smoker & Total \\
\hline Non smoker & 317 & 202 & 157 & 676 \\
\hline Past smoker & 4 & 100 & 6 & 110 \\
\hline
\end{tabular}




\begin{tabular}{|l|c|c|c|c|}
\hline Smoking status \\
\hline \multirow{2}{*}{ ICD-9-CM } & \multicolumn{4}{|c|}{ Electronic medical records } \\
\cline { 2 - 5 } & Non smoker & Past smoker & Current smoker & Total \\
\hline Current smoker & 2 & 30 & 372 & 404 \\
\hline Total & 323 & 332 & 535 & 1,190 \\
\hline
\end{tabular}

\section{Positive predictive value}

Past smoker: 100/110 $=91 \%$

Current smoker: $372 / 404=92 \%$

"Negative" predictive value

Non smoker: $317 / 676=47 \%$

\section{Sensitivity}

Non smoker: $317 / 323=98 \%$

Past smoker: $100 / 332=30 \%$

Current smoker: $372 / 535=70 \%$ 


\section{Key points}

- There may be a difference in the comparative safety of anti-TNF $\alpha$ agents with regard to the risk of serious infections, but little is known about whether the differential risk varies by patient characteristics.

- Our study found that the higher risk of serious infections associated with infliximab might vary by patient age. More studies are needed to confirm or refute this finding. 
Kaiser Permanente Northern California members who had their first dispensing of infliximab or etanercept between $1 / 1 / 1997$ and $12 / 31 / 2007$

$\mathrm{n}=5,208$

Exclude patients younger than 17 years old, without continuous enrollment or pharmacy benefit during the 365-day baseline period preceding the date of first dispensing $n=4,602$

Exclude patients with a diagnosis of serious infections, moderate to severe liver or renal disease, HIV, cancer (except for non-melanoma skin cancer) or solid organ transplantation during the baseline period $n=4,093$

Exclude patients with prior exposure to other biologic disease-modifying anti-rheumatic drugs during the baseline period $\mathrm{n}=\mathbf{3 . 9 2 1}$

Exclude patients with a diagnosis of inflammatory bowel disease $n=3,485$

Figure 1.

Flowchart of the study population, Kaiser Permanente Northern California, 1997-2007 
Table 1

Baseline characteristics of infliximab and etanercept initiators ascertained during the 365-day period before the date of first infliximab or etanercept initiation

\begin{tabular}{|c|c|c|c|}
\hline Characteristics & Infliximab $(n=793)$ & Etanercept $(n=2,692)$ & $\begin{array}{c}\text { Odds ratio }(95 \% \mathrm{CI}) \text { of initiating } \\
\text { infliximab (from the propensity score } \\
\text { model) } a\end{array}$ \\
\hline Mean age (standard deviation) & $57.9(14.1)$ & $50.8(13.0)$ & - \\
\hline \multicolumn{4}{|l|}{ Age in years $(\%)$} \\
\hline $17-45$ & 18.3 & 31.5 & Referent \\
\hline $45-54$ & 23.0 & 29.5 & $1.24(0.95,1.61)$ \\
\hline $55-64$ & 20.8 & 25.1 & $1.11(0.84,1.47)$ \\
\hline$\geq 65$ & 38.0 & 13.9 & $1.73(1.16,2.58)$ \\
\hline Female sex $(\%)$ & 72.0 & 63.4 & $1.07(0.86,1.32)$ \\
\hline \multicolumn{4}{|l|}{ Race/Ethnicity (\%) } \\
\hline Non-Hispanic White & 66.0 & 65.7 & Referent \\
\hline Asian American & 12.1 & 11.1 & $1.41(1.06,1.87)$ \\
\hline African American & 6.7 & 4.7 & $1.55(1.07,2.25)$ \\
\hline Hispanics & 6.1 & 5.4 & $1.51(1.02,2.23)$ \\
\hline Native American & 3.4 & 2.2 & $1.38(0.81,2.36)$ \\
\hline Other/Unknown & 5.8 & 10.9 & $1.01(0.70,1.45)$ \\
\hline \multicolumn{4}{|l|}{ BMI, $\mathrm{kg} / \mathrm{m}^{2}(\%)$} \\
\hline$<18.5$ & 1.9 & 0.9 & $1.37(0.66,2.86)$ \\
\hline $18.5-24$ & 66.2 & 54.5 & Referent \\
\hline $25-29$ & 15.4 & 17.6 & $0.79(0.61,1.02)$ \\
\hline$\geq 30$ & 16.5 & 27.0 & $0.64(0.50,0.82)$ \\
\hline \multicolumn{4}{|l|}{ Smoking status (\%) } \\
\hline Non smoker & 74.3 & 69.9 & Referent \\
\hline Past smoker & 7.8 & 12.1 & $0.60(0.43,0.84)$ \\
\hline Current smoker & 17.9 & 18.0 & $1.20(0.94,1.52)$ \\
\hline $\begin{array}{l}\text { Household in the census block below poverty } \\
\text { line }(\%)\end{array}$ & 18.2 & 13.9 & $1.39(1.10 .1 .77)$ \\
\hline \multicolumn{4}{|l|}{ Type of insurance $(\%)^{b}$} \\
\hline Medicare & 47.0 & 16.0 & $0.77(0.40,1.50)$ \\
\hline Medicaid & 0.9 & 1.1 & $1.06(0.44,2.59)$ \\
\hline Commercial & 55.1 & 85.7 & $0.33(0.18,0.62)$ \\
\hline Private/Self-insured & 39.5 & 12.2 & $2.89(2.30,3.63)$ \\
\hline Other & 0.3 & 0.7 & $0.43(0.10,1.98)$ \\
\hline \multicolumn{4}{|l|}{ Charlson score (\%) } \\
\hline 0 & 16.5 & 33.2 & Referent \\
\hline 1 & 61.3 & 51.5 & $1.30(0.90,1.89)$ \\
\hline$>1$ & 22.2 & 15.3 & $1.50(0.84,2.70)$ \\
\hline \multicolumn{4}{|l|}{ Diagnosis of autoimmune diseases $(\%)^{c}$} \\
\hline Rheumatoid arthritis & 77.2 & 58.8 & $0.55(0.34,0.89)$ \\
\hline
\end{tabular}




\begin{tabular}{|c|c|c|c|}
\hline Characteristics & Infliximab $(n=793)$ & Etanercept $(n=2,692)$ & $\begin{array}{c}\text { Odds ratio }(95 \% \text { CI) of initiating } \\
\text { infliximab (from the propensity score } \\
\text { model }) a\end{array}$ \\
\hline Psoriatic arthritis & 10.8 & 15.8 & $0.91(0.61,1.35)$ \\
\hline Psoriasis & 7.7 & 29.3 & $0.25(0.17,0.37)$ \\
\hline Ankylosing spondylitis & 4.3 & 4.3 & $1.00(0.58,1.74)$ \\
\hline \multicolumn{4}{|c|}{ Diagnosis of other conditions $(\%)^{c}$} \\
\hline Diabetes & 12.4 & 10.5 & $1.11(0.75,1.67)$ \\
\hline COPD & 11.1 & 10.6 & $0.83(0.56,1.23)$ \\
\hline \multicolumn{4}{|c|}{ Medication use in the preceding 365 days (\%) } \\
\hline Methotrexate & 70.5 & 52.9 & $1.94(1.59,2.37)$ \\
\hline Other DMARDs $d$ & 57.1 & 51.2 & $0.94(0.77,1.15)$ \\
\hline NSAIDs & 51.1 & 51.5 & $1.03(0.85,1.24)$ \\
\hline Opioids & 63.8 & 56.5 & $1.23(1.00,1.53)$ \\
\hline Corticosteroids & 77.7 & 74.8 & $1.24(0.98,1.56)$ \\
\hline Antibiotics & 5.9 & 8.0 & $0.71(0.49,1.02)$ \\
\hline$\geq 1$ hospitalization (\%) & 11.1 & 8.7 & $1.15(0.84,1.56)$ \\
\hline \multicolumn{4}{|c|}{ No. of outpatient visits $(\%)^{e}$} \\
\hline $1^{\text {st }}$ quartile & 22.1 & 24.4 & Referent \\
\hline $2^{\text {nd }}$ quartile & 23.0 & 20.2 & $1.17(0.90,1.54)$ \\
\hline $3^{\text {rd }}$ quartile & 29.5 & 29.8 & $0.87(0.65,1.18)$ \\
\hline $4^{\text {th }}$ quartile & 25.5 & 25.6 & $0.88(0.67,1.14)$ \\
\hline \multicolumn{4}{|c|}{ No. of unique medications used $(\%)^{e}$} \\
\hline $1^{\text {st }}$ quartile & 18.3 & 12.9 & Referent \\
\hline $2^{\text {nd }}$ quartile & 33.2 & 31.0 & $0.92(0.66,1.27)$ \\
\hline $3^{\text {rd }}$ quartile & 19.3 & 23.5 & $0.81(0.54,1.23)$ \\
\hline $4^{\text {th }}$ quartile & 29.2 & 32.7 & $0.98(0.67,1.43)$ \\
\hline
\end{tabular}

BMI: Body mass index; CI: Confidence interval; COPD: Chronic obstructive pulmonary disease; DMARDs: Disease-modifying anti-rheumatic drugs; NSAIDs: Non-steroidal anti-inflammatory drugs

${ }^{a}$ Adjusted for all other covariates in the table, as coded in the table

${ }^{b}$ Not mutually exclusive. For each type of insurance, not having that insurance was used as the referent group

${ }^{c}$ For each condition, not having the condition was used as the referent group

$d_{\text {Including hydroxychloroquine, leflunomide, and sulfasalazine }}$

${ }^{e}$ Quartiles were calculated using the entire study cohort 
Table 2

Crude incidence rate of serious infections (per 100 person-years) in initiators of infliximab or etanercept within the first 12 months of initiation, by patient characteristics

\begin{tabular}{|c|c|c|c|}
\hline \multirow[t]{2}{*}{ Characteristics } & \multicolumn{3}{|c|}{ Crude incidence rate ( $95 \%$ confidence interval) } \\
\hline & 0-3 months & 0-6 months & 0-12 months \\
\hline All & $7.5(5.7,9.5)$ & $6.6(5.4,7.9)$ & $5.6(4.8,6.5)$ \\
\hline \multicolumn{4}{|l|}{ Age } \\
\hline$<65$ years & $5.4(3.8,7.5)$ & $4.6(3.5,5.9)$ & $3.9(3.1,4.7)$ \\
\hline$\geq 65$ years & $16.0(10.4,23.4)$ & $14.9(10.9,19.8)$ & $13.0(10.3,16.2)$ \\
\hline \multicolumn{4}{|l|}{ Sex } \\
\hline Female & $8.5(6.3,11.3)$ & $7.2(5.7,9.0)$ & $6.2(5.2,7.4)$ \\
\hline Male & $5.5(3.1,8.9)$ & $5.3(3.6,7.5)$ & $4.4(3.2,5.8)$ \\
\hline \multicolumn{4}{|l|}{ Race/Ethnicity } \\
\hline Non-Hispanic White & $8.5(6.2,11.2)$ & $7.0(5.5,8.8)$ & $5.9(4.9,7.1)$ \\
\hline Asian American & $8.4(3.6,16.6)$ & $7.6(4.1,12.7)$ & $6.5(4.1,9.7)$ \\
\hline African American & $11.5(3.7,26.8)$ & $9.4(4.1,18.6)$ & $8.6(4.7,14.4)$ \\
\hline Native American & $9.6(1.2,34.7)$ & $20.1(8.7,39.7)$ & $14.8(7.4,26.5)$ \\
\hline \multicolumn{4}{|l|}{ Body mass index } \\
\hline$<25 \mathrm{~kg} / \mathrm{m}^{2}$ & $8.8(6.3,11.8)$ & $7.1(5.5,9.0)$ & $6.0(4.9,7.2)$ \\
\hline$\geq 25 \mathrm{~kg} / \mathrm{m}^{2}$ & $5.7(3.5,8.7)$ & $5.9(4.2,8.0)$ & $5.0(3.9,6.4)$ \\
\hline \multicolumn{4}{|l|}{ Smoking status } \\
\hline Non smoker & $6.7(4.8,9.1)$ & $6.1(4.8,7.7)$ & $5.2(4.3,6.2)$ \\
\hline Past smoker & $13.0(6.7,22.8)$ & $10.9(6.6,17.1)$ & $9.4(6.3,13.4)$ \\
\hline Current smoker & $7.2(3.6,12.9)$ & $5.8(3.3,9.2)$ & $5.1(3.4,7.4)$ \\
\hline
\end{tabular}




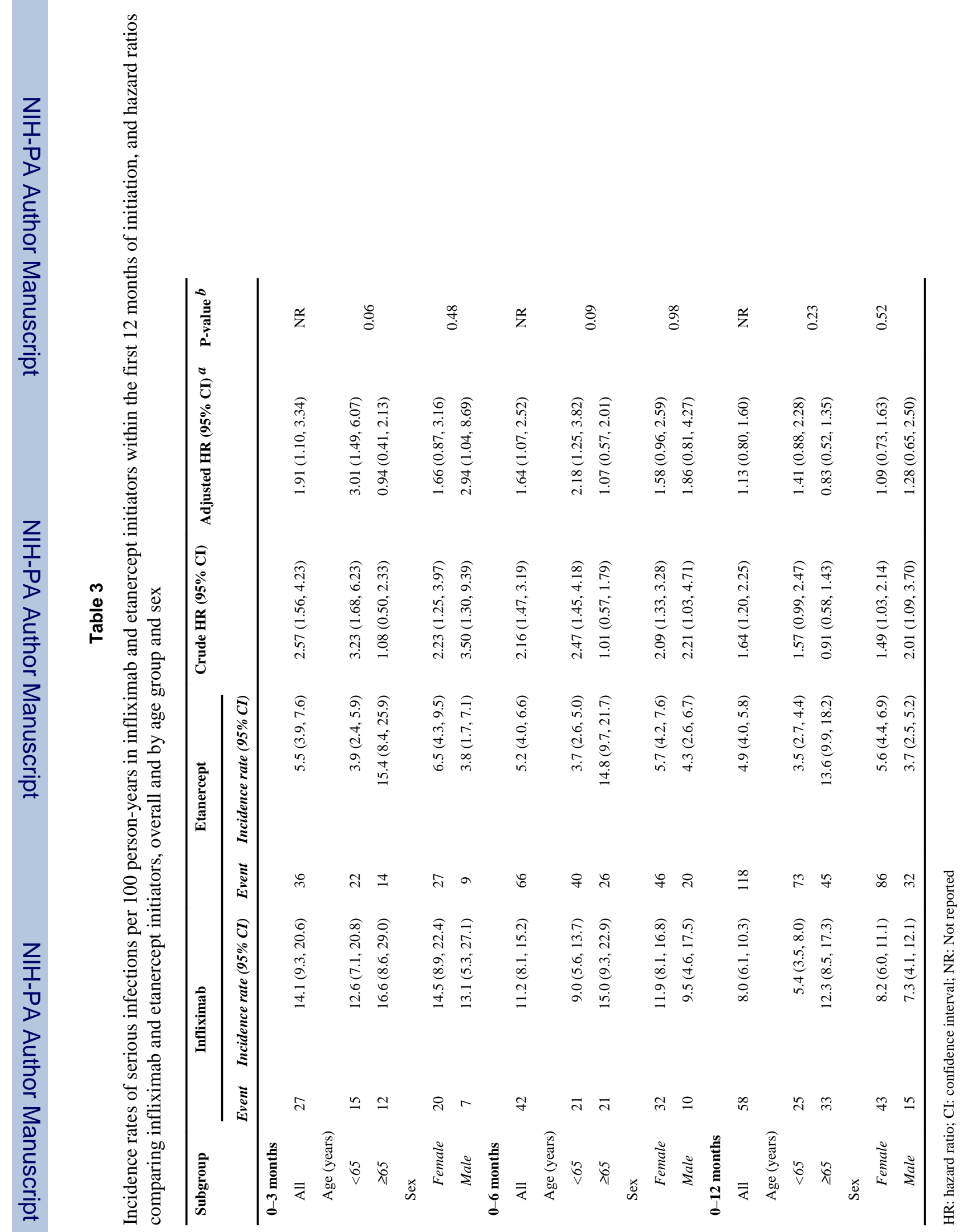




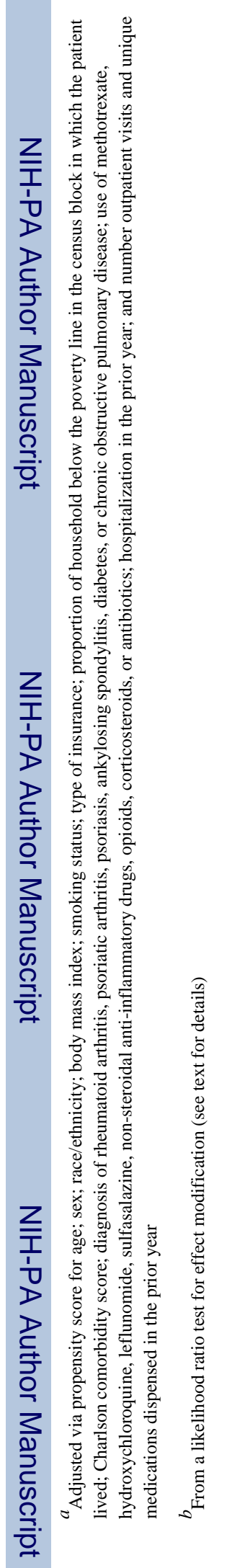

Pharmacoepidemiol Drug Saf. Author manuscript; available in PMC 2013 May 01. 


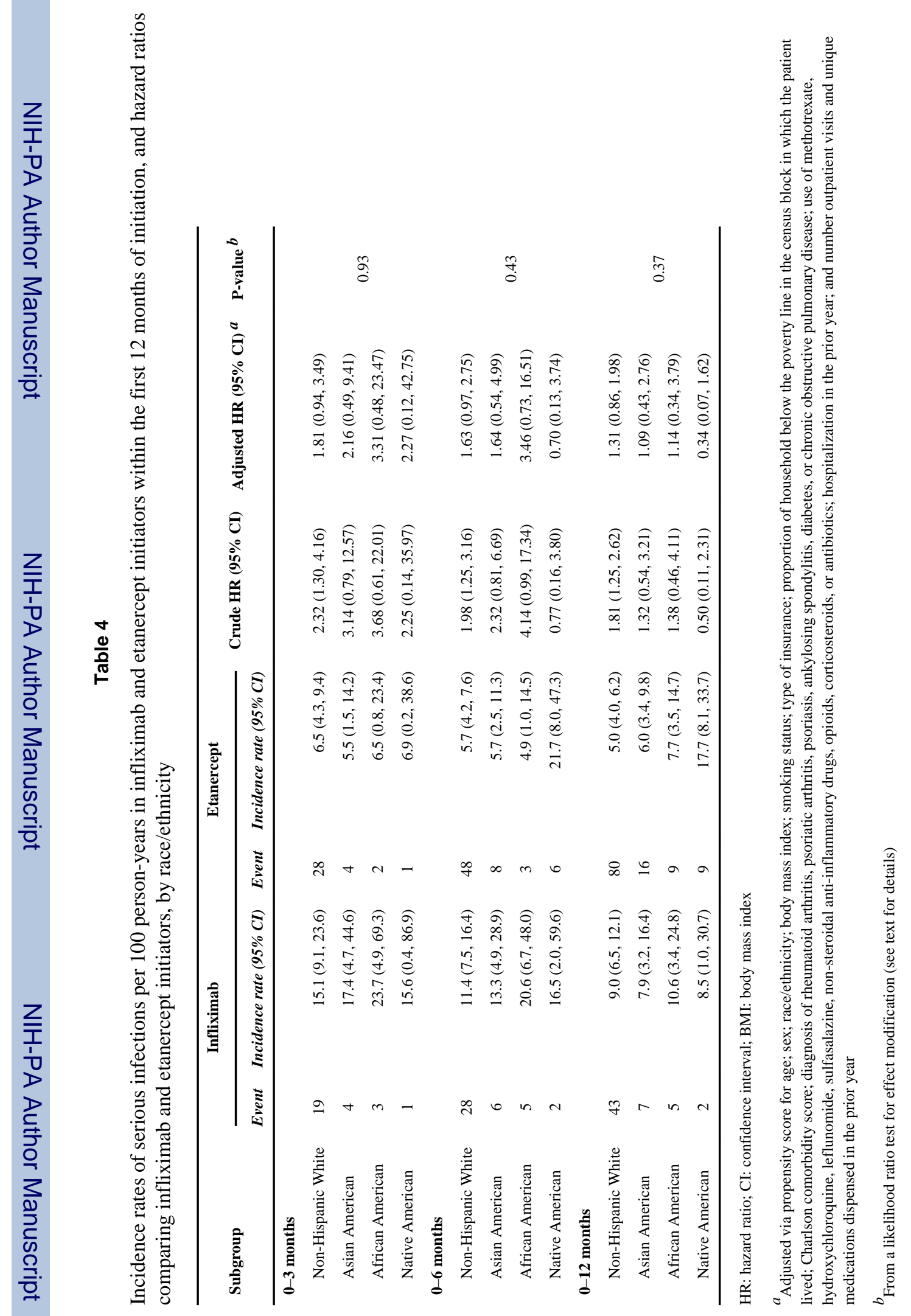

Pharmacoepidemiol Drug Saf. Author manuscript; available in PMC 2013 May 01. 


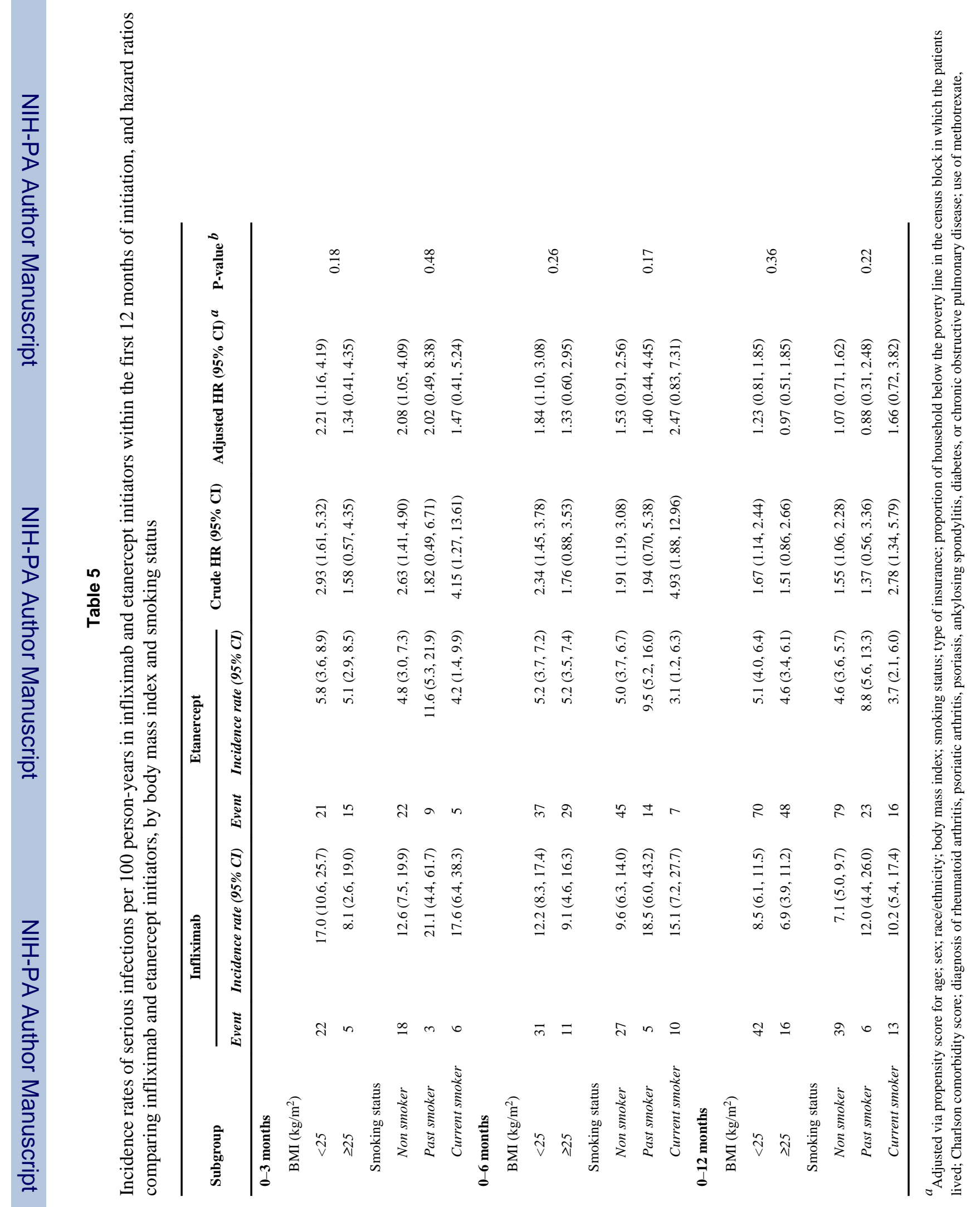


